\title{
Coefficients Estimates of the Class of Biunivalent Functions
}

\author{
Abdullah Aljouiee ${ }^{1}$ and Pranay Goswami ${ }^{2}$ \\ ${ }^{1}$ Mathematics Department, Al-Imam Mohammad Ibn Saud Islamic University, P.O. Box 90189, Riyadh 11613, Saudi Arabia \\ ${ }^{2}$ School of Liberal Studies, Ambedkar University Delhi, Delhi 110006, India
}

Correspondence should be addressed to Abdullah Aljouiee; joal11@gmail.com

Received 29 December 2015; Accepted 21 February 2016

Academic Editor: Gestur Ólafsson

Copyright (C) 2016 A. Aljouiee and P. Goswami. This is an open access article distributed under the Creative Commons Attribution License, which permits unrestricted use, distribution, and reproduction in any medium, provided the original work is properly cited.

Applying the Faber polynomial expansions, we obtain the general coefficient bounds for the class of biunivalent functions with bounded boundary rotations.

\section{Introduction and Definitions}

Let $\mathscr{A}$ be the class of functions $f$ which are analytic in the open unit disk $\mathbb{D}=\{z \in \mathbb{C}:|z|<1\}$ and normalized by the conditions $f(0)=0$ and $f^{\prime}(0)=1$. The Koebe one-quarter theorem [1] ensures that the image of $\mathbb{D}$ under every univalent function $f \in \mathscr{A}$ contains the disk with the center in the origin and the radius of $1 / 4$. Thus, every univalent function $f \in \mathscr{A}$ has an inverse $f^{-1}: f(\mathbb{D}) \rightarrow \mathbb{D}$, satisfying $f^{-1}(f(z))=z(z \in$ $\mathbb{D})$ and

$$
f\left(f^{-1}(w)\right)=w \quad\left(|w|<r_{0}(f) ; r_{0}(f) \leq \frac{1}{4}\right) .
$$

A function $f \in \mathscr{A}$ is said to be biunivalent in $\mathbb{D}$ if both $f$ and $f^{-1}$ are univalent in $\mathbb{D}$, supposing that $\mathbb{D} \subseteq f(\mathbb{D})$, and we denote the class of biunivalent functions by $\sigma$.

In [2], the author defined the following classes $\mathscr{P}_{m}(\beta)$ as follows.

Let $\mathscr{P}_{m}(\beta)(m \geq 2)$ denote the class of univalent analytic functions $h$ that are represented by

$$
\int_{0}^{2 \pi}\left|\frac{\Re P(z)-\alpha}{1-\alpha}\right| d \theta \leq m \pi,
$$

where $z=r e^{i \theta}, k \geq 2$, and $0 \leq \beta<1, z \in \mathbb{D}$.

Lewin [3] investigated the class $\sigma$ of biunivalent functions and obtained the bound for the second coefficient. Brannan and Taha [4] considered certain subclasses of biunivalent functions, similar to the familiar subclasses of univalent functions consisting of strongly starlike, starlike, and convex functions. They introduced the bistarlike functions and the biconvex functions and obtained estimates on the initial coefficients. Recently, Ali et al. [5], Srivastava et al. [6], Frasin and Aouf [7], Goyal and Goswami [8], and many others have introduced and investigated subclasses of biunivalent functions and obtained bounds for the initial coefficients. In the papers of Jahangiri et al. [9] and Hamidi et al. [10], the authors use Faber polynomial to find upper bounds for $a_{n}$. In this paper, we will try to find upper bound of $\left|a_{n}\right|$ for the class $\mathscr{B} \mathscr{R}_{\sigma}^{p}(m ; \beta)$ which is defined below.

Definition 1. A function $f \in \sigma$ is said to be in the class $\mathscr{B} \mathscr{R}_{\sigma}^{p}(m ; \beta)$ if the following conditions are satisfied:

$$
\begin{array}{ll}
\left(f^{\prime}(z)\right)^{p} \in \mathscr{P}_{m}(\beta), & (z \in \mathbb{D}), \\
\left(g^{\prime}(w)\right)^{p} \in \mathscr{P}_{m}(\beta), & (w \in \mathbb{D}),
\end{array}
$$

where $g=f^{-1}$.

Remark 2. Taking $m=2$ in Definition 1, we have a class $\mathscr{B}_{\sigma}(\beta)$ studied by Jahangiri et al. [9].

Using the Faber polynomial expansion [11] of functions $f \in \sigma$ the coefficients of its inverse map $g=f^{-1}$ may be expressed as [12]

$$
g(w)=f^{-1}(w)=w+\sum_{n=2}^{\infty} \frac{1}{n} K_{n-1}^{-n}\left(a_{2}, a_{3} \ldots, a_{n}\right) w^{n},
$$


where

$$
\begin{aligned}
K_{n-1}^{-n} & \\
= & \frac{(-n) !}{(-2 n+1) !(n-1) !} a_{2}^{n-1} \\
& +\frac{(-n) !}{(2(-n+1)) !(n-3) !} a_{2}^{n-3} a_{3} \\
& +\frac{(-n) !}{(-2 n+3) !(n-4) !} a_{2}^{n-4} a_{4} \\
& +\frac{(-n) !}{(2(-n+2)) !(n-5) !} a_{2}^{n-5}\left[a_{5}+(-n+2) a_{3}^{2}\right] \\
& +\frac{(-n) !}{(-2 n+5) !(n-6) !} a_{2}^{n-6}\left[a_{6}+(-2 n+5) a_{3} a_{4}\right] \\
& +\sum_{j \geq 7} a_{2}^{n-j} V_{j},
\end{aligned}
$$

such that $V_{j}$ with $7 \leq j \leq n$ is a homogeneous polynomial in the variables $a_{2}, a_{3}, \ldots, a_{n}$. In particular, the first three terms of $K_{n-1}^{-n}$ are

$$
\begin{aligned}
& \frac{1}{2} K_{1}^{-2}=-a_{2}, \\
& \frac{1}{3} K_{2}^{-3}=3 a_{2}^{2}-a_{3}, \\
& \frac{1}{4} K_{3}^{-4}=-\left(3 a_{2}^{3}-5 a_{2} a_{3}+a_{4}\right) .
\end{aligned}
$$

In general, an expansion of $K_{n}^{p}$ is as [13]

$$
\begin{aligned}
K_{n}^{p}= & p a_{n}+\frac{p !}{(p-2) ! 2 !} D_{n}^{2}+\frac{p !}{(p-3) ! 3 !} D_{n}^{3}+\cdots \\
& +\frac{p !}{(p-n) ! n !} D_{n}^{n},
\end{aligned}
$$

where $D_{n}^{p}=D_{n}^{p}\left(a_{2}, a_{3}, \ldots\right)$ and by [13] or [14],

$$
D_{n}^{l}\left(a_{2}, a_{3}, \ldots\right)=\sum_{l=1}^{\infty} \frac{l !\left(a_{1}\right)^{\mu_{1}} \cdots\left(a_{n}\right)^{\mu_{n}}}{\mu_{1} ! \cdots \mu_{n} !}
$$

while $a_{1}=1$, and the sum is taken over all nonnegative integers $\mu_{1}, \ldots, \mu_{n}$ satisfying evidently $D_{n}^{n}\left(a_{1}, a_{2}, \ldots, a_{n}\right)=a_{1}^{n}$ [12].

The objective of the paper is to find estimates for the coefficients $a_{n}$ for functions in the subclass $\mathscr{B} \mathscr{R}_{\sigma}(m ; \beta)$. These are obtained by employing the techniques used earlier by Jahangiri et al. [9] (see also [10]).

\section{Main Results}

In order to prove our main result for the functions class $f \in$ $\mathscr{B} \mathscr{R}_{\sigma}^{p}(m ; \beta)$, we first use the following lemma.

Lemma 3. Let the function $\Phi$ given by $\Phi(z)=\sum_{n=1}^{\infty} h_{n} z^{n}$ be convex in $\mathbb{D}$. If $\Phi(z) \in \mathscr{P}_{m}(\beta)$, then

$$
\left|h_{n}\right| \leq m(1-\beta) \quad(n \in \mathbb{N}) .
$$

Proof. Proof of this lemma is straightforward, if we write

$$
\begin{aligned}
& \Phi(z)=(1-\beta) p(z)+\beta, \\
& p(z)=1+\sum_{n=1}^{\infty} p_{n} z^{n} \in \mathscr{P}_{m} \\
&=1+(1-\beta) \sum_{n=1}^{\infty} p_{n} z^{n} .
\end{aligned}
$$

This gives

$$
\left|h_{n}\right|=(1-\beta) p_{n}
$$

Using known result [15] for class $\mathscr{P}_{m}$, we have our result.

Theorem 4. Let $f(z)=z+\sum_{n=2}^{\infty} a_{n} z^{n}, z \in \mathbb{D}$, be in the class $\mathscr{B} \mathscr{R}_{\sigma}^{p}(m ; \beta)$ and $a_{k}=0$ for $2 \leq k \leq n-1$; then

$$
\left|a_{n}\right| \leq \frac{m(1-\beta)}{n p} ; \quad n \geq 3(n, m, p \in \mathbb{N}, 0 \leq \beta<1) .
$$

Proof. It is observed that if $\phi(z)=1+\sum_{n=1}^{\infty} \phi_{n} z^{n}$ is an analytic in $\mathbb{D}$, then

$$
(\phi(z))^{p}=1+\sum_{n=1}^{\infty} K_{n}^{p}\left(\phi_{1}, \phi_{2}, \ldots, \phi_{n}\right) z^{n} .
$$

If $f$ is of form (3), then

$$
\left(f^{\prime}(z)\right)^{p}=1+\sum_{n=1}^{\infty} K_{n}^{p}\left(2 a_{2}, 3 a_{3}, \ldots,(n+1) a_{n+1}\right) z^{n} .
$$

Similarly, for $g=f^{-1}$ given by (5), we have

$$
\begin{aligned}
g^{\prime}(w) & =1+\sum_{n=2}^{\infty} K_{n-1}^{-n}\left(a_{2}, a_{3}, \ldots, a_{n}\right) w^{n-1} \\
& =1+\sum_{n=1}^{\infty} b_{n} w^{n} .
\end{aligned}
$$

Consequently, for $\left(g^{\prime}(w)\right)^{p}$, we have

$$
\left(g^{\prime}(w)\right)^{p}=1+\sum_{n=1}^{\infty} K_{n}^{p}\left(b_{1}, b_{2}, \ldots, b_{n}\right) w^{n} .
$$

Now from Definition 1 , there exist two functions $p(z)$ and $q(w)$ that belong to $\mathscr{P}(m ; \beta)$ such that

$$
\begin{aligned}
& \left(f^{\prime}(z)\right)^{p}=p(z)=1+c_{1} z+c_{2} z^{2}+\cdots, \\
& \left(g^{\prime}(w)\right)^{p}=q(w)=1+d_{1} w+d_{2} w^{2}+\cdots .
\end{aligned}
$$

Now comparing the coefficients of (15) and (18), the following is given:

$$
K_{n-1}^{p}\left(2 a_{2}, 3 a_{3}, \ldots, n a_{n}\right)=c_{n-1} .
$$


Similarly, from (17) and (19),

$$
K_{n-1}^{p}\left(b_{1}, b_{2}, \ldots, b_{n-1}\right)=d_{n-1} .
$$

If $a_{k}=0$ for $2 \leq k \leq n-1$, then we can combine (20) and (21) and using relation (8), it yields

$$
\begin{aligned}
& n p a_{n}=c_{n-1}, \\
& p b_{n-1}=-n p a_{n}=d_{n-1} .
\end{aligned}
$$

Now taking absolute value in both sides of above equations and using Lemma 3, we get

$$
\begin{aligned}
& \left|a_{n}\right| \leq \frac{\left|c_{n-1}\right|}{n p}=\frac{\left|d_{n-1}\right|}{n p}=\frac{m(1-\alpha)}{n p}, \\
& \quad(n, m, p \in \mathbb{N}, n \geq 3) .
\end{aligned}
$$

If we relax the condition $a_{k}=0$ in Theorem 4 , we have the following theorem.

Theorem 5. Let $f(z)=z+\sum_{n=2}^{\infty} a_{n} z^{n}, z \in \mathbb{D}$, be in the class $\mathscr{B} \mathscr{R}_{\sigma}^{p}(m ; \beta)$; then

$$
\begin{aligned}
\text { (i) }\left|a_{2}\right| \leq \frac{m(1-\beta)}{p} ; & \\
\text { (ii) }\left|a_{3}-a_{2}^{2}\right| \leq \frac{2 m(1-\beta)}{3 p} ; & \\
& (n, p \in \mathbb{N}, 0 \leq \beta<1) .
\end{aligned}
$$

Proof. Since $f \in \mathscr{B} \mathscr{R}_{\sigma}^{p}(m ; \beta)$, from (3) and (4) we have

$$
\begin{aligned}
& \left(f^{\prime}(z)\right)^{p}=p(z), \\
& \left(g^{\prime}(w)\right)^{p}=q(w),
\end{aligned}
$$

where $p(z) \in \mathscr{P}_{m}(\beta)$ and $q(w) \in \mathscr{P}_{m}(\beta)$. It is easy to see that the functions $p$ and $q$ have the following Taylor expansions:

$$
\begin{aligned}
& p(z)=1+c_{1} z+c_{2} z^{2}+c_{3} z^{3}+\cdots, \quad z \in \mathbb{D}, \\
& q(w)=1+d_{1} w+d_{2} w^{2}+d_{3} w^{3}+\cdots, \quad w \in \mathbb{D} .
\end{aligned}
$$

Now, equating the coefficients in (25), we get

$$
\begin{aligned}
2 p a_{2} & =c_{1}, \\
-2 p a_{2} & =d_{1} ;
\end{aligned}
$$

taking absolute value in both equations and using Lemma 3, $\left|a_{2}\right| \leq m(1-\beta) / n p$ is given.

Further, from (25) and (8), it follows that

$$
\begin{aligned}
2 p(p-1) a_{2}^{2}+3 p a_{3} & =c_{1}, \\
\frac{p(p-1)}{2} b_{1}^{2}+p b_{2} & =2 p(p+2) a_{2}^{2}-3 p a_{3}=d_{2} .
\end{aligned}
$$

Subtracting (29) from (28), we get

$$
6 p\left(a_{3}-a_{2}^{2}\right)=c_{2}-d_{2}
$$

Now taking absolute value in both sides, we will get desired result.

Remark 6. If we put $m=2$ in Theorems 4 and 5 , we get the result obtained by Jahangiri et al. [9].

\section{Competing Interests}

The authors declare that there are no competing interests regarding the publication of this paper.

\section{References}

[1] P. L. Duren, Univalent Functions, vol. 259 of Grundlehren der Mathematischen Wissenschaften, Springer, New York, NY, USA, 1983.

[2] K. S. Padmanabhan and R. Parvatham, "Properties of a class of functions with bounded boundary rotation," Annales Polonici Mathematici, vol. 31, no. 3, pp. 311-323, 1975.

[3] M. Lewin, "On a coefficient problem for bi-univalent functions," Proceedings of the American Mathematical Society, vol. 18, pp. 63-68, 1967.

[4] D. A. Brannan and T. S. Taha, "On some classes of biunivalent functions," Studia Universitatis Babes-Bolyai Series Mathematica, vol. 31, no. 2, pp. 70-77, 1986.

[5] R. M. Ali, S. K. Lee, V. Ravichandran, and S. Supramaniam, "Coefficient estimates for bi-univalent Ma-Minda starlike and convex functions," Applied Mathematics Letters, vol. 25, no. 3, pp. 344-351, 2012.

[6] H. M. Srivastava, A. K. Mishra, and P. Gochhayat, "Certain subclasses of analytic and bi-univalent functions," Applied Mathematics Letters, vol. 23, no. 10, pp. 1188-1192, 2010.

[7] B. A. Frasin and M. K. Aouf, "New subclasses of bi-univalent functions," Applied Mathematics Letters, vol. 24, no. 9, pp. 15691573, 2011.

[8] S. P. Goyal and P. Goswami, "Estimate for initial Maclaurin coefficients of bi-univalent functions for a class defined by fractional derivatives," Journal of the Egyptian Mathematical Society, vol. 20, no. 3, pp. 179-182, 2012.

[9] J. M. Jahangiri, S. G. Hamidi, and S. A. Halim, "Coefficient estimates for a class of meromorphic bi-univalent functions," Comptes Rendus Mathematique, vol. 351, no. 9-10, pp. 349-352, 2013.

[10] S. G. Hamidi, S. A. Halim, and J. M. Jahangiri, "Coefficients of bi-univalent functions with positive real part derivatives," The Bulletin of the Malaysian Mathematical Society Series, vol. 37, no. 3, pp. 633-640, 2014.

[11] G. Faber, "Über polynomische Entwickelungen," Mathematische Annalen, vol. 57, no. 3, pp. 389-408, 1903.

[12] H. Airault and A. Bouali, "Differential calculus on the Faber polynomials," Bulletin des Sciences Mathématiques, vol. 20, no. 3, pp. 179-222, 2006.

[13] H. Airault, "Symmetric sums associated to the factorization of Grunsky coefficients," in Proceedings of the Groups and Symmetries, Montreal, Canada, April 2007. 
[14] M. Schiffer, "Faber polynomials in the theory of univalent functions," Bulletin of the American Mathematical Society, vol. 54, pp. 503-517, 1948.

[15] B. Pinchuk, "Functions of bounded boundary rotation," Israel Journal of Mathematics, vol. 10, pp. 6-16, 1971. 


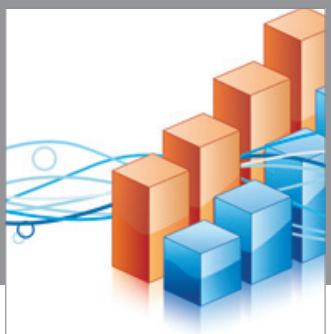

Advances in

Operations Research

vatem alat4

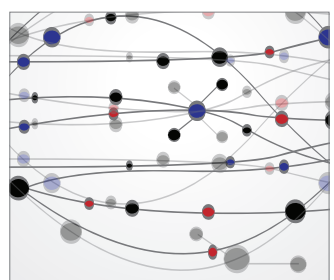

\section{The Scientific} World Journal
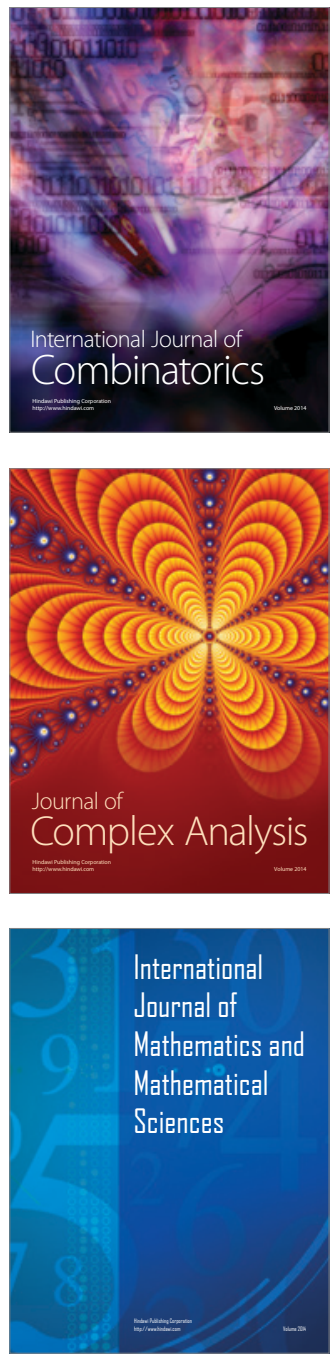
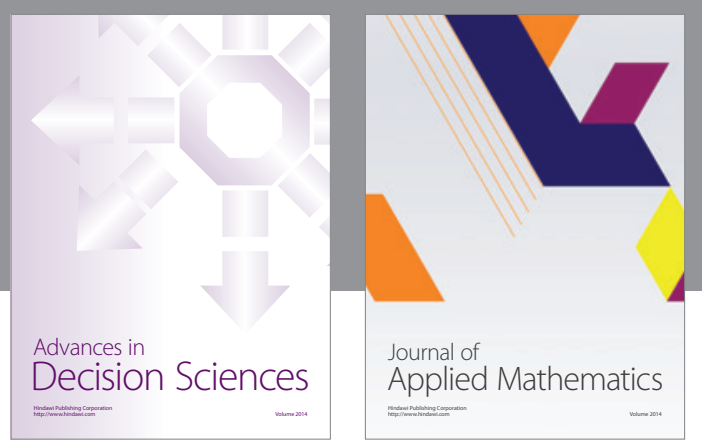

Algebra

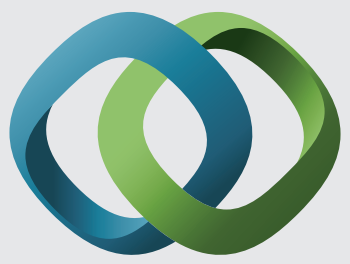

\section{Hindawi}

Submit your manuscripts at

http://www.hindawi.com
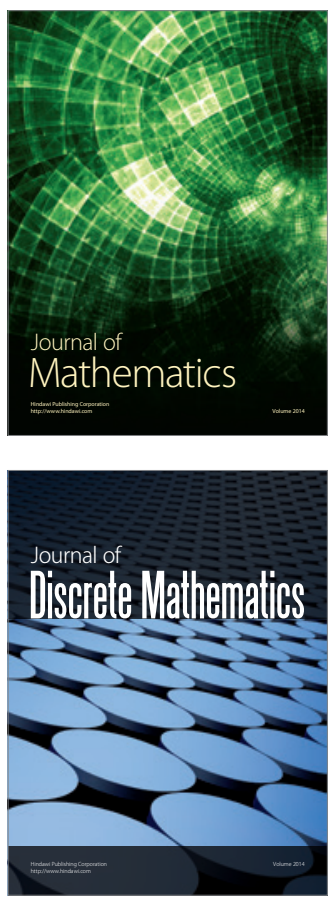

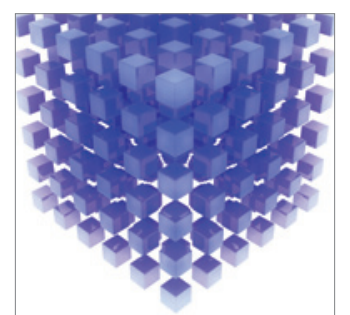

Mathematical Problems in Engineering
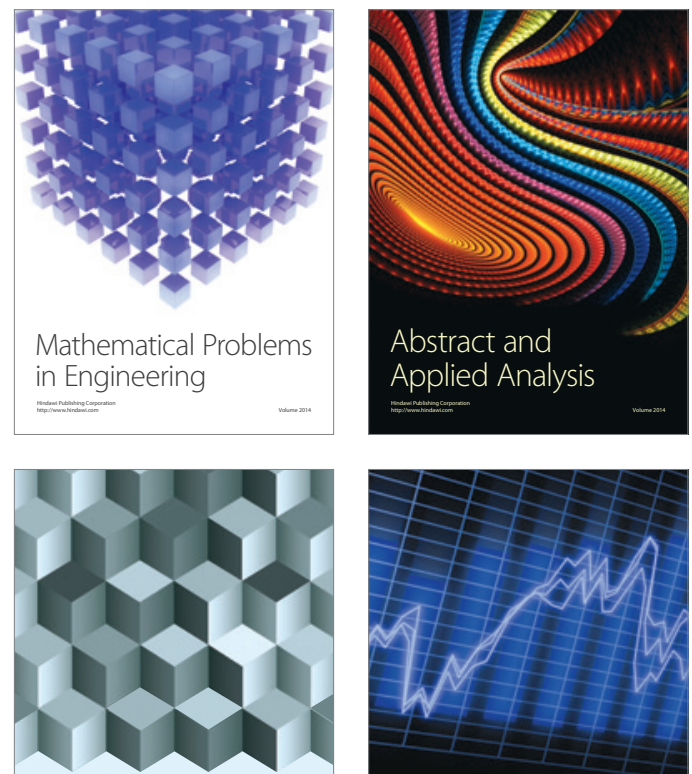

Journal of

Function Spaces

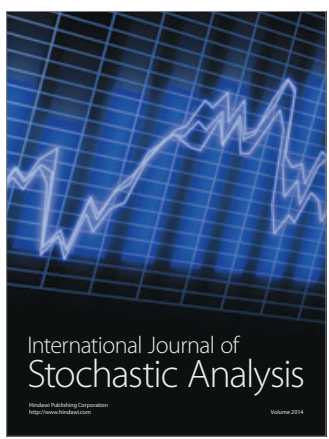

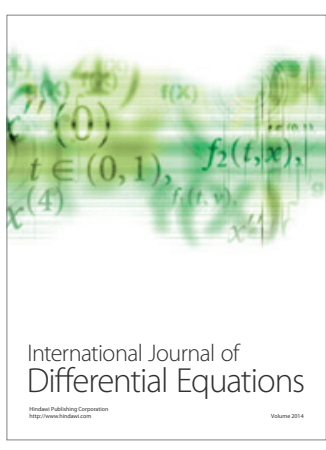
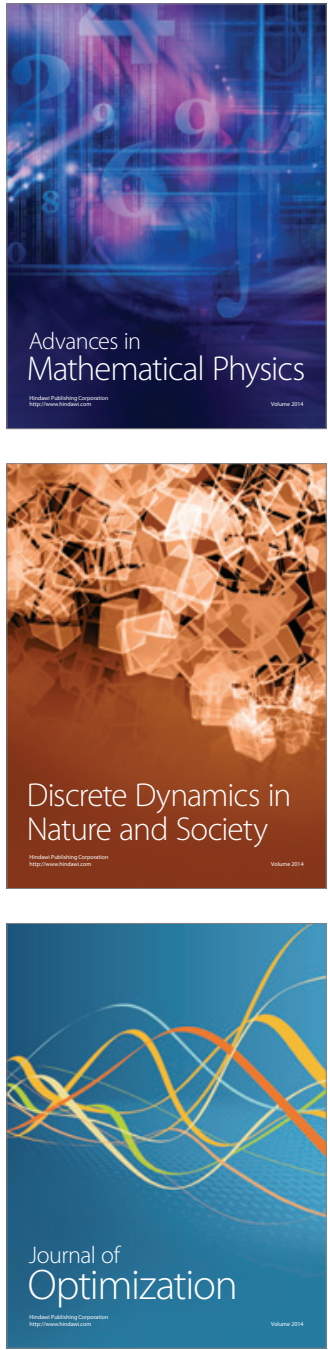